\title{
The Prophylactic and Therapeutic Effects of Saffron Extract and Crocin on Ethanol Withdrawal Syndrome in Mice
}

\author{
Maryam Shoja', Soghra Mehri ${ }^{1,2,3}$, Bahareh Amin ${ }^{4}$, Vahid Reza Askari ${ }^{5}$, Hossein \\ Hosseinzadeh ${ }^{1,2 *}$
}

'Department of Pharmacodynamics and Toxicology, School of Pharmacy, Mashhad University of Medical Sciences, Mashhad, Iran ${ }^{2}$ Pharmaceutical Research Center, Pharmaceutical Technology Institute, Mashhad University of Medical Sciences, Mashhad, Iran ${ }^{3}$ Division of Neurocognitive Sciences, Psychiatry and Behavioral Sciences Research Center, Mashhad University of Medical Sciences, Mashhad, Iran

${ }^{4}$ Cellular and Molecular Research Center, Department of Physiology and Pharmacology, Faculty of Medicine, Sabzevar University of Medical Sciences, Sabzevar, Iran

${ }^{5}$ Department of Pharmacology, Faculty of Medicine, Mashhad University of Medical Sciences, Mashhad, Iran

\section{Key Words}

alcohol withdrawal, pentylenetetrazol, Crocus sativus, crocin, open field test

\begin{abstract}
Objectives: Ethanol withdrawal following its chronic use is a serious outcome and challenging to treatment. The chronic use of ethanol induces a progressive neuroplasticity in different reigns of brain. In this study we evaluated the effects of aqueous extract of Crocus sativus L. (saffron) and its active compound, crocin, on the withdrawal behavior induced after repeated administration of ethanol, in two regimens of prophylactic (administration of drugs concomitant with the induction of dependence) and treatment (administration of drugs during the period of ethanol withdrawal) in mice which received ethanol.
\end{abstract}

Methods: Ethanol dependence was induced by oral administration of $10 \% \mathrm{v} / \mathrm{v}$ ethanol $(2 \mathrm{~g} / \mathrm{kg})$ for 7 days. The aqueous extracts of saffron (40,80 and 160) and crocin $(10,20$ and $40 \mathrm{mg} / \mathrm{kg})$ were administered to mice in two regimens of prophylactic (along with ethanol) and treat-

Received: Oct 31, 2017 Reviewed: Oct 25, 2018 Accepted: Nov 14, 2018

(1) This is an Open-Access article distributed under the terms of the Creative Common Attribution Non-Commercial License (http://creativecommons.org/licenses/by-nc/4.0/) which permits unrestricted noncommercial use, distribution, and reproduction in any medium, provided the original work is properly cited.

( This paper meets the requirements of KS X ISO 9706, ISO 9706-1994 and ANSI/NISO Z39.48-1992 (Permanence of Paper). ment (during withdrawal period). Diazepam ( $1 \mathrm{mg} / \mathrm{kg})$ was used as a positive control. Six hours after discontinuation of the ethanol, seizure was evaluated by the sub-convulsive dose of pentyleneltetrazole (PTZ) (30 $\mathrm{mg} / \mathrm{kg}$ ). The open field test and Rota rod test were used for evaluation of locomotor activity and motor incoordination, respectively.

Results: Both extracts and crocin increased the number of crossed lined in the open field test. PTZ kindling seizure was inhibited in animals received extract (80 and $160 \mathrm{mg} / \mathrm{kg}$ ) in both regimens. Motor incoordination was only improved following administration of crocin.

Conclusion: The aqueous extract of saffron and crocin can be considered as safe agents and reliable alternative to diazepam in management of ethanol withdrawal syndrome.

\section{Introduction}

Addiction is a chronic and relapsing disturbance which is reflected in an individual pathologically pursuing reward and relief by substance use. This disturbance leads to dysfunction in the biological, psycho-

"Corresponding Author

Hossein Hosseinzadeh. Department of Pharmacodynamics and Toxicology, School of Pharmacy, Mashhad University of Medical Sciences, Mashhad 1365-91775, Iran. Tel: +98-51-3180-1193 Fax: +98-51-3882-3251

Email: hossenzadehh@mums.ac.ir 
logical and social manifestations and also contributes to loss of control over drug intake and dysfunctional emotional responses such as dysphoria, anxiety and irritability that explain a motivational withdrawal syndrome subsequent to drug barricade [1].

Ethanol and drug dependence are important public health problems worldwide. The outbreak of ethanol and drug dependence in the United States and other parts of the world is really significant [2]. One of the problems of chronic ethanol consumption is physical dependence which resulting in physical withdrawal responses after stop taking of ethanol. The mechanisms which are involved in tolerance, dependence and withdrawal syndrome following chronic ethanol consumption are complex. Ethanol has depressant effects on the central nervous system (CNS) and when large amounts of ethanol are used over time the brain adapts to this depressant effect, in this way, stimulate a level of tolerance [3]. In proportion to the duration of a recent drinking habit and the amount of ethanol consumed, clinical symptoms of alcohol withdrawal syndrome (AWS) occur with varying intensity [4]. Different studies have shown that benzodiazepines, such as diazepam, are the perfect choice for the treatment of withdrawal syndrome, because it can modulate symptoms of seizures and anxiety. Although, dependence to these drugs can be mentioned as important problem in treatment of ethanol dependence [5].

Crocus sativus L., universally known as saffron, is a plant cultivated in different parts of the world and has traditional uses $[6,7]$ and multiple pharmacological properties $[8$, 9]. Saffron contains several constituents such as safranal (accountable for saffron odor and aroma), picrocrocin (responsible for saffron bitter flavor) and crocin (the main saffron antioxidant as a dye agent) [10]. Saffron or its main component crocin have been exhibited valuable effects including cardioprotective [11, 12] hepatoprotective [13-15], neuroprotective [16, 17] antidote against different toxins [18], antinociceptive [19], anti-inflammatory [20] and antitumor [21] properties. Additionally effect of saffron in digestive disorder [22], metabolic syndrome [18] and erectile dysfunction [23] has been reported. Regarding to safety of saffron and crocin $[24,25]$ different clinical applications of them have been mentioned in various disorders [26].

Interestingly, saffron or crocin could change the chemical neurotransmitters such as dopamine in the brain, [27] and showed several effects on nervous system [28] including, antidepressant [29], anticonvulsant [30], memory enhancer [31,32], hypnotic [33] and attenuation of morphine withdrawal syndrome [34].

The aim of this study was to determine the prophylactic and therapeutic effect of saffron extract and crocin on ethanol-induced withdrawal syndrome and dependence in mice. It should be noted that the study focused on the effects of saffron extract and crocin on the important symptoms of ethanol withdrawal syndrome including decrease the seizure threshold and motor impairment. Anticonvulsant, antidepressant and anxiolytic effects of saffron extract and crocin will help us to evaluate their efficiency in treatment of ethanol withdrawal as these compounds does not induce any dependence in comparison to benzodiazepines.

\section{Material and Methods}

\subsection{Chemicals}

Diazepam ampul (10 mg/2 ml, Caspian Tamin, Iran), Pentylenetetrazol (Sigma-Aldrich), ethanol 96\% (Taghtir Khorasan, Iran) were used in our study.

\subsection{Crocin preparation}

Stigmas of Crocus. sativus L. were purchased from Novin Saffron (collected from Ghaen, Khorasan province, Northeast of Iran). Crocin was extracted and purified according to the method which previously described [35].

\subsection{Preparation of extract}

$30 \mathrm{~g}$ of stigma powder was soaked in $1500 \mathrm{ml}$ water for 72 hours. The mixture of plant and water was subsequently centrifuged (5 minutes, 3000 RPM) and was placed overnight in the freezer $\left(-20^{\circ} \mathrm{C}\right)$. Then, the extract was dried in Freeze dryer [33].

\subsection{Animals}

Male mice weighing (25 - $35 \mathrm{~g}$ ) used in the study. The animals were housed in colony rooms with $\left(21 \pm 2^{\circ} \mathrm{C}\right)$ under a 12/12 hours light/dark cycle. Animals had free access to food and water. The experiments were approved by with Mashhad University of Medical Sciences, Ethical Committee Acts $(\mathrm{No}=922237)$.

\subsection{Experimental Design}

The mice ( $\mathrm{n}=7$ per group) were divided into 18 groups which described as follows:

Group 1- Control group treated with water by gavage (negative control)

Group 2- Ethanol alone received animals (2 g/kg ethanol as the $10 \%(\mathrm{v} / \mathrm{v})$ solution by gavage) [36].

\section{(Prophylactic groups)}

Group 3- Animals received ethanol + diazepam (1 mg/ $\mathrm{kg}$, IP) [36].

Group 4, 5, 6- Animals received ethanol + aqueous saffron extract $(40,80,160 \mathrm{mg} / \mathrm{kg}$ in groups $4,5,6$ respectively) [37].

Group 7, 8, 9- Animals received ethanol $+\operatorname{crocin}(10,20$, $40 \mathrm{mg} / \mathrm{kg}$ in groups $7,8,9$ respectively) [11].

Group 10- Aqueous saffron extract alone administered animals at the highest dose (160 mg/kg) Group 11- Crocin alone administered animals at the highest dose $(40 \mathrm{mg} / \mathrm{kg})$

In mentioned groups, for induction dependence, ethanol was administrated once daily for 7 days. Withdrawal symptoms for ethanol were evaluated 6 hours after the last administration of ethanol. Additionally, diazepam, aqueous saffron extract or crocin were administrated IP once daily for 7 days and 6 hours after the last administration 
of ethanol.

\section{(Treatment groups)}

Group 12- Animals received ethanol + diazepam (1 mg/ $\mathrm{kg}, \mathrm{IP})$ [36].

Group 13, 14, 15- Animals received ethanol + aqueous saffron extract $(40,80,160 \mathrm{mg} / \mathrm{kg}$. Group 16, 17, 18- Animals received ethanol + crocin $(10,20,40 \mathrm{mg} / \mathrm{kg})$.

In treatment groups a single dose of diazepam, aqueous saffron extract or crocin was administrated 6 hours after the last administration of ethanol. In both (prophylactic and treatment groups) ethanol withdrawal symptoms were evaluated using open field test, Rota rod test and PTZ Kindling.

\subsection{Open field test (OFT)}

The apparatus for this test, made of white wood, had a floor of $45 \times 45 \mathrm{~cm}$ divided by lines into 25 squares of 9 $\times 9 \mathrm{~cm}$. The walls, $40-\mathrm{cm}$ high, were also painted in white. Each mouse was placed in the middle of the apparatus and its behavior was observed for 10 minutes. The total number of squares crossed was evaluated [38].

\subsection{Rota rod test}

Rota rod test includes a rotating bar that the animal is placed on it. In this test total time each animal was able to maintain its balance walking on top of the rod was recorded. The speed of the Rota rod accelerated from 10 to 20 rpm over a 20 seconds period. The maximum time the animal remains on the device, 300 seconds was considered. In this method, 24 hours before the test, mice were trained on the apparatus and only the mice that have the ability to maintain on the rotating rod at least 30 seconds were used [39].

\subsection{PTZ Kindling}

To induce kindling, a subconvulsant dose of pentylenetetrazole $(30 \mathrm{mg} / \mathrm{kg}$, IP) was injected 6 hours after the last dose of ethanol. After the PTZ injection, the animals were observed for the occurrence of convulsive behavior for 30 minutes. Animals entering into convulsion have shown convulsive state which include ear and facial jerks, myoclonic body jerks without upright position, myoclonic jerks, upright position with bilateral forelimb clonus, clonic-tonic seizures and generalised clonic-tonic seizures and loss of postural control [36].

\subsection{Statistical Analysis}

The results were expressed as mean \pm SD $(n=7)$. The statistical analysis was performed by One-way ANOVA followed by Tukey test in the Open field and Rota rod test and Fisher exact test were used in the PTZ. In all three tests $p$ value at $<0.05$ was considered as statistically significant.

\section{Results}

\subsection{Open field test}

\subsection{Effect of aqueous saffron extract and crocin on locomotor activity in ethanol withdrawal mice model in prophylactic groups}

Administration of ethanol decreased the number of lines crossed during the open field test as compared to negative control $(\mathrm{P}<0.001)$. Administration of diazepam, the extracts $(40,80$ and $160 \mathrm{mg} / \mathrm{kg})$ or crocin $(10,20$ and $40 \mathrm{mg} /$ $\mathrm{kg}$ ) once a day during induction of ethanol dependence significantly increased animal total locomotion in comparison to ethanol-treated group (figure 1).

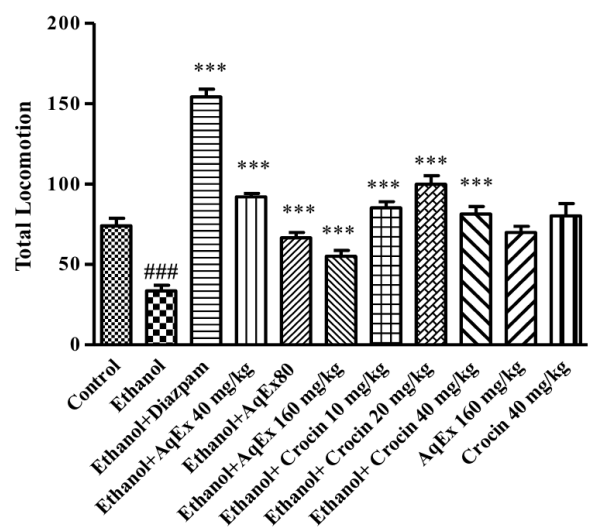

Figure 1 Prophylactic effect of aqueous saffron extracts $(40,80,160 \mathrm{mg} / \mathrm{kg})$ and crocin $(10,20,40 \mathrm{mg} / \mathrm{kg})$ during open field test in ethanol withdrawal mice model. Bars represent mean $\pm \mathrm{SD}(\mathrm{n}=7)$. \#\#\#P $<0.001$ vs control, ${ }^{* * *} \mathrm{P}$ $<0.001$ vs ethanol group. The statistical analysis was performed by One-way ANOVA followed by Tukey test. AqEx = aqueous saffron extract.

\subsubsection{Effect of aqueous saffron extract and crocin on locomotor activity in ethanol withdrawal mice model in treatment groups}

As shown in figure 2, in ethanol-treatment animals the number of lines crossed during the open field test markedly reduced as compared to negative control $(\mathrm{P}<0.001)$, while single dose administration of diazepam, the extracts (40, 80 and $160 \mathrm{mg} / \mathrm{kg}$ ) or crocin $(10,20$ and $40 \mathrm{mg} / \mathrm{kg}$ ) could improve locomotor activity in comparison to ethanol group $(\mathrm{P}<0.001)$.

\subsection{Rota rod test}

\subsubsection{Effect of aqueous saffron extract and crocin on motor coordination in ethanol withdrawal mice model in prophylactic groups}

The findings from the Rota rod test demonstrated that following ethanol withdrawal, motor coordination signifi- 


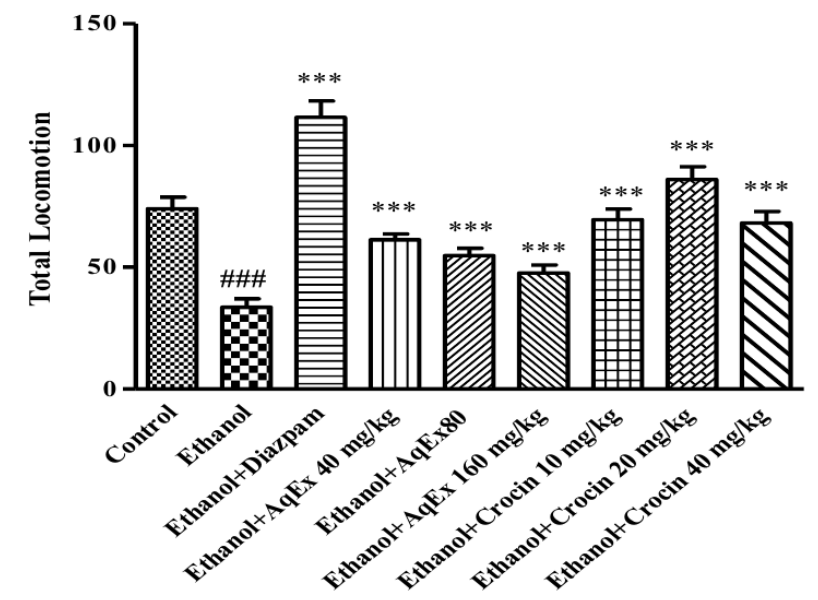

Figure 2 Therapeutic effect of aqueous saffron extracts $(40,80,160 \mathrm{mg} / \mathrm{kg})$ and crocin $(10,20,40 \mathrm{mg} / \mathrm{kg})$ during open field test in ethanol withdrawal mice model. Bars represent mean $\pm \mathrm{SD}(\mathrm{n}=7)$. \#\#\#P $<0.001$ vs control, ${ }^{* * *} \mathrm{P}$ $<0.001$ vs ethanol group. The statistical analysis was performed by One-way ANOVA followed by Tukey test. AqEx $=$ aqueous saffron extract.

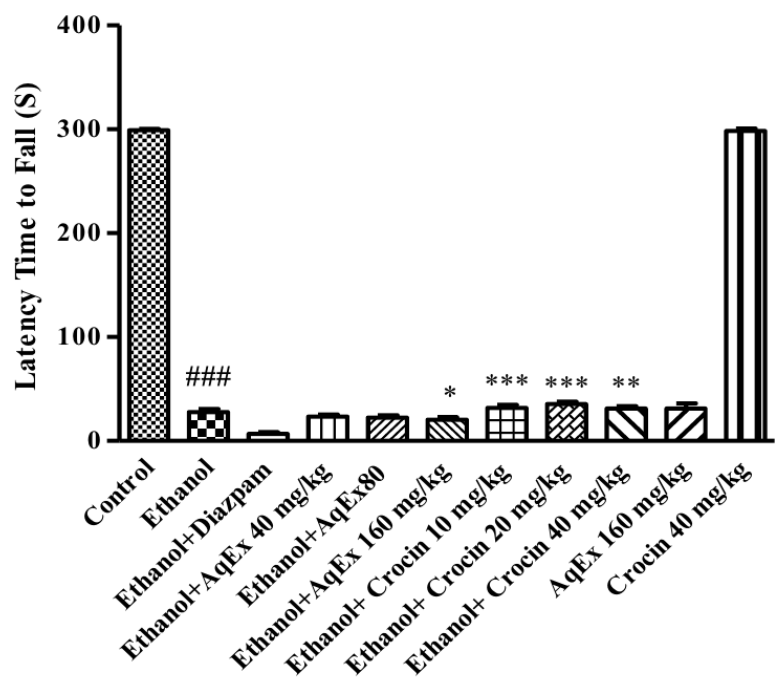

Figure 3 Prophylactic effect of aqueous saffron extracts $(40,80,160 \mathrm{mg} / \mathrm{kg})$ and crocin $(10,20,40 \mathrm{mg} / \mathrm{kg})$ during Rotarod test in ethanol withdrawal mice model. Bars represent mean $\pm \mathrm{SD}(\mathrm{n}=7)$. \#\#\#P $<0.001$ vs control, ${ }^{*} \mathrm{P}<0.05$, ${ }^{* *} \mathrm{P}<0.01,{ }^{* * *} \mathrm{P}<0.001$ vs ethanol group. The statistical analysis was performed by One-way ANOVA followed by Tukey test. AqEx = aqueous saffron extract. cantly diminished $(\mathrm{P}<0.001$ vs control group). Treatment of animals with crocin $(10,20$ and $40 \mathrm{mg} / \mathrm{kg})$ during induction of ethanol dependence improved the performance of mice in rota rod test. Administration of aqueous saffron extract in doses of 40 and $80 \mathrm{mg} / \mathrm{kg}$ could not change motion balance when compared to ethanol group (figure 3).

\subsubsection{Effect of aqueous saffron extract and crocin on motor coordination in ethanol withdrawal mice model in treatment groups}

As shown in figure 4, single dose administration of diazepam, the extracts $(40,80$ and $160 \mathrm{mg} / \mathrm{kg}$ ) or crocin (10, 20 and $40 \mathrm{mg} / \mathrm{kg}$ ) didn't recover mice performance in Rota rod test.

\subsection{PTZ Kindling}

\subsubsection{Effect of aqueous saffron extract and crocin on PTZ Kindling in ethanol withdrawal mice model in prophylactic groups}

Administration of sub-convulsive dose of PTZ $(30 \mathrm{mg} /$ kg i.p) in negative control group has not induced any convulsions, whereas in ethanol withdrawal mice, $100 \%$ convulsion was observed following PTZ administration. As shown in figure 5 , treatment of animals with diazepam $(1 \mathrm{mg} / \mathrm{kg})$ or aqueous saffron extract $(40,80,160 \mathrm{mg} / \mathrm{kg})$ markedly attenuated the percentage of convulsion $(\mathrm{P}<$ 0.001 vs ethanol group), while crocin in all doses could not decrease seizure threshold.

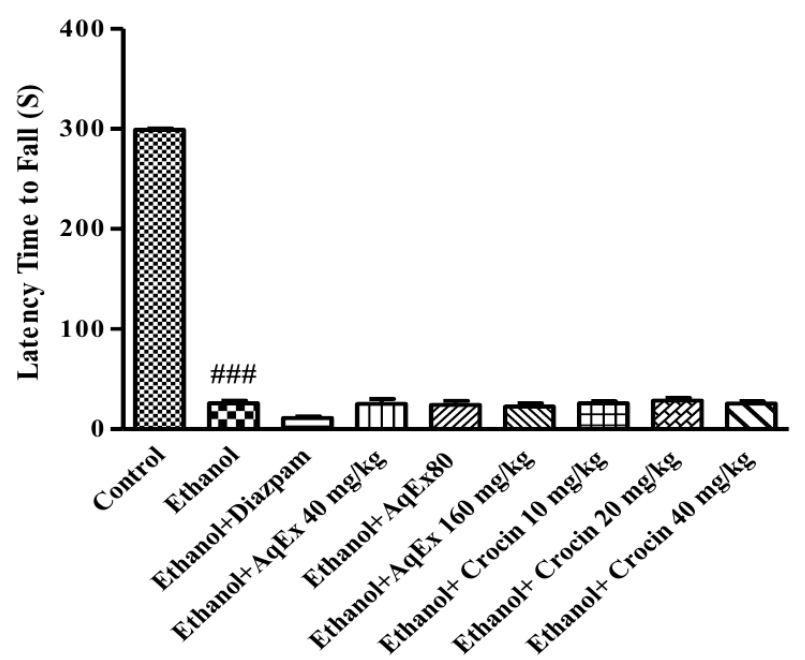

Figure 4 Therapeutic effect of aqueous saffron extracts $(40,80,160 \mathrm{mg} / \mathrm{kg})$ and crocin $(10,20,40 \mathrm{mg} / \mathrm{kg})$ during rotarod test in ethanol withdrawal mice model in treatment groups. Bars represent mean $\pm \mathrm{SD}(\mathrm{n}=7)$.

$\# \# \#$ P $<0.001$ vs control. The statistical analysis was performed by One-way ANOVA followed by Tukey test. AqEx = aqueous saffron extract. 


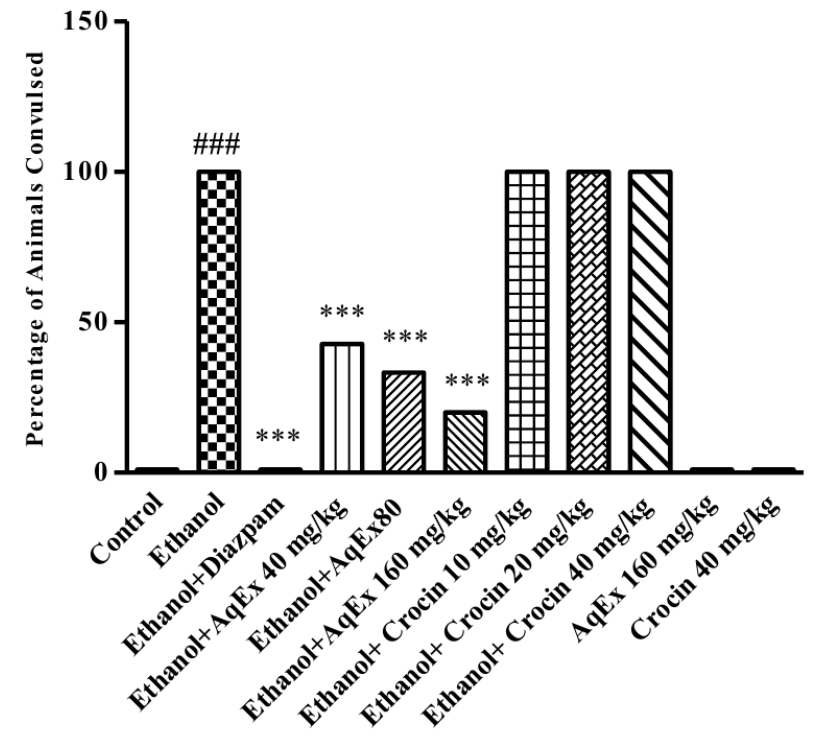

Figure 5 Prophylactic effect of aqueous saffron extracts (40, 80, $160 \mathrm{mg} / \mathrm{kg})$ and crocin (10, 20, $40 \mathrm{mg} / \mathrm{kg}$ ) during PTZ test in ethanol withdrawal mice model. Bars represent mean $\pm \mathrm{SD}(\mathrm{n}=7)$. \#\#\#P $<0.001$ vs control, ${ }^{* * *} \mathrm{P}<0.001$ vs ethanol group. The statistical analysis was performed by Fisher exact test. AqEx = aqueous saffron extract.

\subsubsection{Effect of aqueous saffron extract and crocin on PTZ Kindling in ethanol withdrawal mice model in treatment groups}

According to results which have been shown in figure 6 , single dose administration of diazepam ( $1 \mathrm{mg} / \mathrm{kg}$ ), or aqueous saffron extracts $(80 \mathrm{mg} / \mathrm{kg} \mathrm{P}<0.01,160 \mathrm{mg} / \mathrm{kg} \mathrm{P}$ $<0.001$ ) significantly increased seizure threshold in comparison to ethanol-received animals. However, crocin in all doses was not effective in control of convulsion during ethanol withdrawal period.

\section{Discussion}

In this study, the prophylactic and therapeutic effect of saffron extract and crocin on ethanol-induced withdrawal syndrome and dependence in mice were examined. Both extract and crocin increased the number of crossed lined of withdrawn animals in the open field test. PTZ kindling seizure was inhibited in animals treated with aqueous extract (40, 80 and $160 \mathrm{mg} / \mathrm{kg}$ ). Administration of crocin but not extract improved motor incoordination in animals.

Ethanol withdrawal syndrome occurs when a heavy drinker suddenly stops or significantly reduces his ethanol use. This syndrome includes psychological symptoms and physical signs. The results of animal studies have been shown, administration of ethanol with multiple periodic

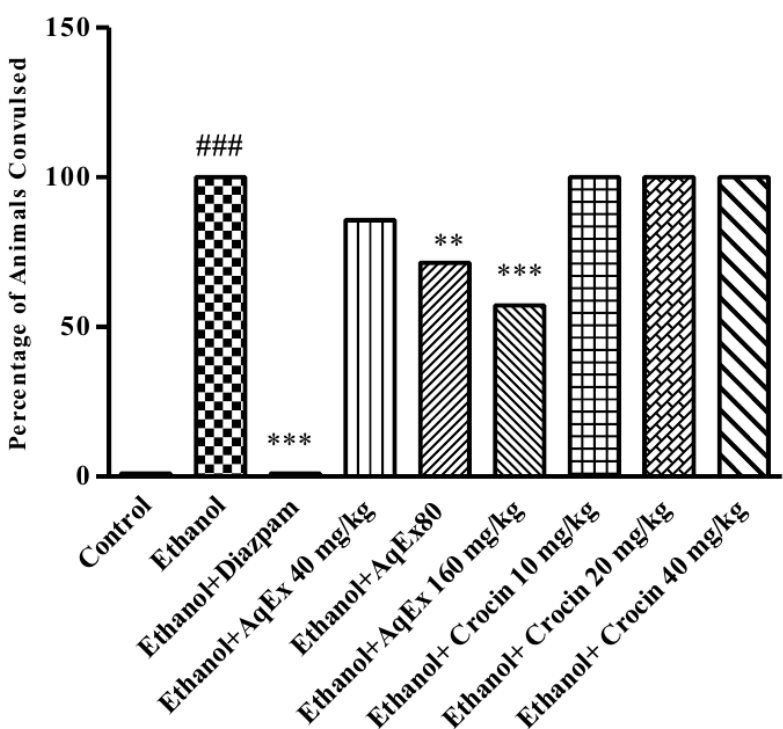

Figure 6 Therapeutic effect of aqueous saffron extracts $(40,80,160 \mathrm{mg} / \mathrm{kg})$ and crocin $(10,20,40 \mathrm{mg} / \mathrm{kg})$ during PTZ test in ethanol withdrawal mice model. \#\#\#P $<0.001$ vs control, ${ }^{* *} \mathrm{P}<0.01,{ }^{* * *} \mathrm{P}<0.001$ vs ethanol group. The statistical analysis was performed by Fisher exact test. AqEx = aqueous saffron extract.

pattern lead to more consecutive signs of withdrawal [40].

In previous studies it was found that saffron extracts and their constituents, crocin and safranal have serotonergic, noradrenergic and dopaminergic activity [29]. In withdrawal process the level of dopamine and serotonin neurotransmitters significantly change. [41]. Therefore, it can be concluded saffron extracts and its constituent crocin based on effects on these mediators may have advantages in the treatment of ethanol withdrawal.

Ethanol withdrawal leads to reduction of animal exploratory behaviors in open field test. This reduction often resulted from withdrawal anxiety, which can be explained by decrease in number of lines crossed by animals. The results of this test in the current study exhibited the number of lines crossing activity by mice significantly increased following treatment with aqueous saffron extract $(40,80,160$ $\mathrm{mg} / \mathrm{kg})$ and crocin $(10,20,40 \mathrm{mg} / \mathrm{kg})$ in both prophylactic and treatment groups similar to diazepam. These data can be due to anxiolytic activity of aqueous saffron extract and crocin. In the previous research Hosseinzadeh et al (2009) [33] showed that the aqueous saffron extract had an anxiolytic effect in mice which was comparable to diazepam. This finding was obtained in the elevated plus-maze test. Since GABA agonist has anti-anxiety properties [42], the beneficial effects of saffron extract and crocin in this study may be mediated in part through GABA-mimetic effects. Although more mechanistic studies should be done.

The NMDA receptors and glutamatergic system are partly responsible for the anxiety-like behavior seen during ethanol withdrawal [43]. Based on Berger et al. study (2011) 
[44], effect on NMDA receptor may be involved in the antagonistic effect of saffron extract on ethanol withdrawal syndrome. Also, pervious results suggested that crocin antagonized the inhibitory effect of ethanol on NMDA receptor-mediated responses in hippocampal neurons [45].

According to the results of the Rota rod test, administration of crocin $(10,20$ and $40 \mathrm{mg} / \mathrm{kg})$ significantly increased motion balance in prophylactic models. In the previous study [33], saffron showed muscle relaxation and motor imbalance effects while these effects were not observed during administration of crocin.

In the treatment groups, there were no significant differences between the aqueous saffron extract (40, 80 and $160 \mathrm{mg} / \mathrm{kg}$ ) and crocin (10, 20 and $40 \mathrm{mg} / \mathrm{kg}$ ) groups for motion balance as compared to ethanol group. To achieve this different mechanism and effect, further studies are needed.

In this study, administration of diazepam $(1 \mathrm{mg} / \mathrm{kg})$ or aqueous saffron extracts ( $80 \mathrm{mg} / \mathrm{kg}$ and $160 \mathrm{mg} / \mathrm{kg}$ ) significantly increased seizure threshold in comparison to ethanol-received animals. It can be concluded that aqueous saffron extract and diazepam have similar antiepileptic effects in PTZ model of epilepsy. It seems safranal is responsible for the antiepileptic effects of saffron extract. According to previous studies, safranal exhibited an anticonvulsant activity in PTZ-induced seizures while crocin did not show anticonvulsant activity $[30,46]$. Also, antiabsence properties of safranal in acute experimental seizure models have been reported [47].

Our data suggested that crocin was not involved in the anticonvulsant effects of saffron stigma in current study.

\section{Conclusion}

This study exhibited that the aqueous extract of saffron and crocin may have possible benefit in the treatment of ethanol withdrawal and can be used as a safe agent and a reliable alternative to benzodiazepines.

\section{Acknowledgment}

The authors are thankful to the Vice Chancellor of Research, Mashhad University of Medical Sciences for financial support. Results of current study are part of MSc thesis.

\section{Conflict of interest}

The authors declare that there are no conflicts of interest.

\section{References}

1. Koob GF. Addiction is a reward deficit and stress surfeit disorder. Front Psychiatry. 2013;4:72.

2. Rahman S, Engleman EA, Bell RL. Nicotinic receptor modulation to treat alcohol and drug dependence. Front Neurosci. 2015;8:426.

3. Smith-Alnimer M, Watford MF. Alcohol Withdrawal and Delirium Tremens: Fast recognition may save a patient from the worst of withdrawal. Am J Nurs.
2004;104(5):72-75.

4. Bayard M, McIntyre J, Hill KR, Woodside J. Alcohol Withdrawal Syndrome. Am Fam Physician. 2004;69(6):14431450.

5. Ennaceur A, Michalikova S, van Rensburg R, Chazot PL. Tolerance, sensitization and dependence to diazepam in Balb/c mice exposed to a novel open space anxiety test. Behav Brain Res. 2010;209(1):154-164.

6. Mollazadeh H, Emami SA, Hosseinzadeh H. Razi's Al-Hawi and saffron (Crocus sativus): a review. Iran J Basic Med Sci. 2015;18(12):1153-1166.

7. Hosseinzadeh H, Nassiri-Asl M. Avicenna's (Ibn Sina) the Canon of Medicine and Saffron (Crocus sativus): A Review. Phytother Res. 2013;27(4):475-483.

8. Alavizadeh SH, Hosseinzadeh H. Bioactivity assessment and toxicity of crocin: a comprehensive review. Food Chem Toxicol. 2014;64:65-80.

9. Rameshrad M, Razavi BM, Hosseinzadeh H. Saffron and its derivatives, crocin, crocetin and safranal: a patent review. Expert Opin Ther Pate. 2017;1-19.

10. Ríos JL, Recio MC, Giner RM, Máñez S. An update review of saffron and its active constituents. Phytother Res. 1996;10(3):189-193.

11. Razavi M, Hosseinzadeh H, Abnous K, Motamedshariaty VS, Imenshahidi M. Crocin restores hypotensive effect of subchronic administration of diazinon in rats. Iran J Basic Med Sci. 2013;16(1):64-72.

12. Mehdizadeh R, Parizadeh MR, Khooei AR, Mehri S, Hosseinzadeh H. Cardioprotective Effect of Saffron Extract and Safranal in Isoproterenol-Induced Myocardial Infarction in Wistar Rats. Iran J Basic Med Sci. 2013;16(1):56-63.

13. Lari $\mathrm{P}$, Abnous K, Imenshahidi M. Evaluation of diazinon-induced hepatotoxicity and protective effects of crocin. Toxicol Ind Health. 2013;31(4):367-376.

14. Hassani FV, Mehri S, Abnous K, Birner-Gruenberger R, Hosseinzadeh H. Protective effect of crocin on BPA-induced liver toxicity in rats through inhibition of oxidative stress and downregulation of MAPK and MAPKAP signaling pathway and miRNA-122 expression. Food Chem Toxicol. 2017;107:395-405.

15. Yousefsani BS, Pourahmad J, Hosseinzadeh H. The mechanism of protective effect of crocin against liver mitochondrial toxicity caused by arsenic III. Toxicol Mech Meth. 2018;28(2):105-114.

16. Mohammadzadeh L, Hosseinzadeh $\mathrm{H}$, Abnous $\mathrm{K}$, Razavi BM. Neuroprotective potential of crocin against malathion-induced motor deficit and neurochemical alterations in rats. Environ Sci Pollut Res Int. 2018;25(5):4904-4914.

17. Heidari S, Mehri S, Hosseinzadeh H. Memory enhancement and protective effects of crocin against D-galactose aging model in the hippocampus of Wistar rats. Iran J Basic Med Sci. 2017;20(11):1250-1259.

18. Razavi BM, Hosseinzadeh H. Saffron: a promising natural medicine in the treatment of metabolic syndrome. J Sci Food Agric. 2017;97(6):1679-1685.

19. Amin B, Hosseinzadeh H. Evaluation of aqueous and ethanolic extracts of saffron, Crocus sativus L., and its constituents, safranal and crocin in allodynia and hyperalgesia induced by chronic constriction in- 
jury model of neuropathic pain in rats. Fitoterapia. 2012;83(5):888-895.

20. Hosseinzadeh H, Younesi HM. Antinociceptive and anti-inflammatory effects of Crocus sativus L. stigma and petal extracts in mice. BMC Pharmacol. 2002;2:1-8.

21. Rastgoo M, Hosseinzadeh H, Alavizadeh H, Abbasi A, Ayati Z, Jaafari MR. Antitumor Activity of PEGylated Nanoliposomes Containing Crocin in Mice Bearing C26 Colon Carcinoma. Planta Med. 2013;79(06):447451.

22. Khorasany A, Hosseinzadeh H. Therapeutic effects of saffron (Crocus sativus L.) in digestive disorders: a review. Iran J Basic Med Sci. 2016;19(5):455-469.

23. Shamsa A, Hosseinzadeh H, Molaei M, Shakeri MT, Rajabi O. Evaluation of Crocus sativus L. (saffron) on male erectile dysfunction: A pilot study. Phytomedicine. 2009;16(8):690-693.

24. Mohamadpour AH, Ayati Z, Parizadeh MR, Rajbai O, Hosseinzadeh H. Safety Evaluation of Crocin (a constituent of saffron) Tablets in Healthy Volunteers. Iran J Basic Med Sci. 2013;16(1):39-46.

25. Bostan H, Mehri S, Hosseinzadeh H. Toxicology effects of saffron and its constituents: a review. Iran J Basic Med Sci. 2017;20(2):110-121.

26. Moshiri M, Vahabzadeh M, Hosseinzadeh H. Clinical Applications of Saffron (Crocus sativus) and its Constituents: A Review. Drug Res. 2015;65(06):287-295.

27. Ettehadi H, Mojabi SN, Ranjbaran M, Shams J, Sahraei H, Hedayati M, et al. Aqueous Extract of Saffron (Crocus sativus) Increases Brain Dopamine and Glutamate Concentrations in Rats. J Behav Brain Sci. 2013;3:315319.

28. Nassiri-Asl M, Hosseinzadeh H. Chapter 3 - Neuropharmacology Effects of Saffron (Crocus sativus) and Its Active Constituents A2 - Watson, Ronald Ross, in Bioactive Nutraceuticals and Dietary Supplements in Neurological and Brain Disease, V.R. Preedy, Editor. 2015;Academic Press:San Diego.29-39.

29. Hosseinzadeh H, Karimi G, Niapoor M. Antidepressant effect of Crocus sativus L. stigma extracts and their constituents, crocin and safranal, in mice. J Med Plants. 2004;3(11):48-58.

30. Hosseinzadeh H, Khosravan V. Anticonvulsant effects of aqueous and ethanolic extracts of Crocus sativus L. stigmas in mice. Arc Iran Medi. 2002;5:44-47.

31. Amin B, Malekzadeh M, Heidari MR, Hosseinzadeh H. Effect of Crocus sativus extracts and its active constituent safranal on the harmaline-induced tremor in mice. Iran J Basic Med Sci. 2015;18(5):449-458.

32. Ghadrdoost B, Vafaei AA, Rashidy-Pour A, Hajisoltani R, Bandegi AR, Motamedi F, et al. Protective effects of saffron extract and its active constituent crocin against oxidative stress and spatial learning and memory deficits induced by chronic stress in rats. Eur J Pharmacol. 2011;667(1):222-229.

33. Hosseinzadeh H, Noraei NB. Anxiolytic and hypnotic effect of Crocus sativus aqueous extract and its constituents, crocin and safranal, in mice. Phytother Res. 2009;23(6):768-774.

34. Hosseinzadeh H, Jahanian Z. Effect of crocus sativus L. (saffron) stigma and its constituents, crocin and safra- nal, on morphine withdrawal syndrome in mice. Phytother Res. 2010;24(5):726-730.

35. Hadizadeh F, Mohajeri S, Seifi M. Extraction and purification of crocin from saffron stigmas employing a simple and efficient crystallization method. Pak J Biol Sci. 2010;13:691-698.

36. Ruby B, Benson MK, Kumar EP, Sudha S, Wilking JE. Evaluation of Ashwagandha in alcohol withdrawal syndrome. Asian Pac J Trop Dis. 2012;2:856-860.

37. Hassani FV, Naseri V, Razavi BM, Mehri S, Abnous K, Hosseinzadeh H. Antidepressant effects of crocin and its effects on transcript and protein levels of CREB, BDNF, and VGF in rat hippocampus. Daru. 2014;22:19.

38. Pardon MC, Pérez-Diaz F, Joubert C, Cohen-Salmon C. Age-dependent effects of a chronic ultramild stress procedure on open-field behaviour in B6D2F1 female mice. Physiol Behav. 2000;70(1):7-13.

39. McIlwain KL, Merriweather MY, Yuva-Paylor LA, Paylor R. The use of behavioral test batteries: effects of training history. Physiolo Behav. 2001;73(5):705-717.

40. Knapp DJ, Breese GR. Models of chronic alcohol exposure and dependence. Psychiatric Disorders: Methods Protoc. 2012;205-230.

41. Uzbay IT, Usanmaz SE, Tapanyigit EE, Aynacioglu S, Akarsu ES. Dopaminergic and serotonergic alterations in the rat brain during ethanol withdrawal: association with behavioral signs. Drug Alcohol Depend. 1998;53(1):39-47.

42. Treit D, Engin E, McEown K. Animal models of anxiety and anxiolytic drug action. Curr Top Behav Neurosci. 2010;2:121-60.

43. Kyzar EJ, Pandey SC. Molecular mechanisms of synaptic remodeling in alcoholism. Neurosci Lett. 2015;601:11-19.

44. Berger F, Hensel A, Nieber K. Saffron extract and trans-crocetin inhibit glutamatergic synaptic transmission in rat cortical brain slices. Neuroscience. 2011;180:238-247.

45. Abe K, Sugiura M, Shoyama Y, Saito H. Crocin antagonizes ethanol inhibition of NMDA receptor-mediated responses in rat hippocampal neurons. Brain Res. 1998;787(1):132-138.

46. Hosseinzadeh H, Talebzadeh F. Anticonvulsant evaluation of safranal and crocin from Crocus sativus in mice. Fitoterapia. 2005;76(7):722-724.

47. Sadeghnia HR, Cortez MA, Liu D, Hosseinzadeh H, Snead OC. Antiabsence effects of safranal in acute experimental seizure models: EEG and autoradiography. J Pharm Pharm Sci. 2008;11(3):1-14. 